\title{
CALIBRAÇÃO DO SENSOR CAPACITIVO EC-5 EM UM LATOSSOLO EM FUNÇÃO DA DENSIDADE DO SOLO
}

Elson de Jesus Antunes Júnior ${ }^{1}$, José Alves Junior ${ }^{2}$ \& Derblai Casaroli ${ }^{3}$

1 - Engenheiro agrícola, doutorando em Agronomia UFG/Goiânia-GO, elson.j.jr@gmail.com

2 - Engenheiro agrônomo, professor da UFG/Goiânia-GO, josealvesufg@yahoo.com.br

3 - Engenheiro agrônomo, professor da UFG/Goiânia-GO, derblaicasaroli@yahoo.com.br

\section{Palavras-chave:}

instrumentação agrícola manejo da água no solo permissividade dielétrica sensor FDR

umidade do solo

\section{RESUMO}

A determinação do conteúdo de água no solo, de forma não destrutiva e instantânea, facilita o manejo da água no solo, bem como o entendimento da dinâmica de água no mesmo. O estudo teve como objetivo calibrar o sensor capacitivo $\mathrm{ECH}_{2} \mathrm{O}$ EC-5 (Decagon) para um Latossolo Vermelho distrófico em diferentes densidades. O sensor foi calibrado em laboratório para as densidades do solo: 1,$10 ; 1,20 ; 1,25 ; 1,30$ e $1,35 \mathrm{Mg} \mathrm{m}^{-3}$. O solo foi saturado e seco em estufa à $40{ }^{\circ} \mathrm{C}$ em intervalos de 2 horas, esperando-se 1 hora para que as amostras resfriassem à temperatura ambiente. Em cada intervalo, o conteúdo de água foi determinado por gravimetria e pelo sensor. Os resultados demonstraram que a equação de calibração padrão do fabricante superestimou em $38,4 \%$ o conteúdo de água no solo. No entanto, depois de calibrado, para o solo em questão, o sensor apresentou alta acurácia na estimativa do conteúdo de água do solo. Os resultados demonstraram ainda que a densidade do solo influencia na calibração dos sensores. Portanto, para obtenção do conteúdo de água no solo nas diferentes densidades, devese corrigir os dados coletados em campo, para o solo em questão, pela seguinte equação: $\theta \mathrm{e}$ $=0,652376 \theta \mathrm{o}+2,12808 \rho \mathrm{s}^{2}-5,2077 \rho \mathrm{s}+3,19237$. Esta equação apresenta o coeficiente de determinação igual a 0,9488 e, para o pior caso, uma acurácia média de $0,024 \mathrm{~m}^{3} \mathrm{~m}^{-3}$.

\section{CALIBRATION OF THE EC-5 CAPACITIVE SENSOR IN AN OXISOL DUE TO SOIL DENSITY}

\section{ABSTRACT}

In a non-destructive and instantaneous way, the determination of the water content in the soil facilitates the water management in the soil, as well as the understanding of the water dynamics in the soil. The objective of the study was to calibrate the $\mathrm{ECH}_{2} \mathrm{O}$ EC-5 (Decagon) capacitive sensor for a dystrophic Red Oxisol at different densities. The sensor was calibrated in the laboratory for soil densities: $1.10 ; 1.20 ; 1.25 ; 1.30$ and $1.35 \mathrm{Mg} \mathrm{m}^{-3}$. The soil was saturated and oven dried at $40{ }^{\circ} \mathrm{C}$ at 2 hours intervals, waiting 1 hour for the samples to cool to room temperature. At each interval, the water content was determined by gravimetry and by the sensor. The results showed that the manufacturer's standard calibration equation overestimates the soil water content by $38.4 \%$. However, after calibration, for this soil, the sensor presented high accuracy in the estimation of soil water content. The results also demonstrated that the soil density influences the calibration of the sensors. Therefore, in order to obtain soil water content at different densities, the data collected in the field should be corrected for this soil by the following equation: $\theta \mathrm{e}=0.652376 \theta \mathrm{o}+2.12808 \rho \mathrm{s}^{2}-5.2077 \rho \mathrm{s}+3.19237$. This equation presents the coefficient of determination equal to 0.9488 and, for the worst case, an average accuracy of $0.024 \mathrm{~m}^{3} \mathrm{~m}^{-3}$. 


\section{INTRODUÇÃO}

O monitoramento da umidade do solo na camada de interesse em tempo real é essencial para o bom manejo da irrigação, bem como, para aplicações em outros processos hidrológicos, tais como: alocação e movimento de água no solo e cálculos do balanço hídrico. A água no solo é um dos elementos fundamentais para o crescimento e desenvolvimento das plantas e transporte de solutos (FARES et al., 2015). Devido à dificuldade na obtenção da umidade do solo em escala espacial e temporal para aplicações hidrológicas, climáticas e agrícolas, foram desenvolvidos diferentes métodos para essa obtenção (MINET et al., 2012).

Os métodos são classificados como diretos ou indiretos (ROMANO, 2014). O método direto (padrão) consiste em secar a amostra a $105^{\circ} \mathrm{C}$, até obtenção de massa constante, sendo este o método gravimétrico que é utilizado principalmente na pesquisa e como referência para calibração de outros métodos. Enquanto que os métodos indiretos utilizam sensores do tipo resistência do solo, tensiômetros, técnica dielétrica do solo, como: reflectometria no domínio do tempo (TDR), reflectometria no domínio da frequência (FDR) ou capacitivos, técnica da capacitância do solo e sensores de fluxo térmico da umidade do solo (SU et al., 2014).

Há no mercado uma grande variedade de sensores no que diz respeito ao princípio de funcionamento e os mais utilizados são os capacitivos, pois apresentam baixo custo, fácil operação e fundamentam-se no valor da permissividade dielétrica $(\varepsilon)$ do meio (BOGENA et al., 2007). No caso do solo, este valor se encontra entre 2 5, o ar é próximo de 1 e a água apresenta alta permissividade, ou seja, 80, assim é possível obter-se a umidade volumétrica (DOBSON et al., 1985). A equação utilizada para tal conversão foi determinada por TOPP et al. (1980). No entanto, estes sensores apresentam variabilidade quanto à salinidade, textura e densidade do solo. Assim, se faz necessária a calibração dos sensores para sua correta utilização.

A Decagon apresenta uma versatilidade de produtos, dentre os sensores de determinação da umidade do solo está o $\mathrm{ECH}_{2} \mathrm{O}$ EC-5, sendo o mais recente sensor desenvolvido pela empresa e opera à frequência de $70 \mathrm{MHz}$ (Decagon Devices Inc., 2016). Seu design com duas hastes curtas facilita a inserção do sensor no solo, porém reduz o volume de solo explorado pelo sensor (BOGENA et al., 2007).

De acordo com ROSENBAUM et al. (2011), o sensor $\mathrm{ECH}_{2} \mathrm{O}$ EC-5 apresenta como vantagem o fácil manuseio, robustez, possuir um sistema de baixo consumo de energia e baixo custo de aquisição, quando comparado a outros equipamentos no mercado. Além de realizar mensurações instantâneas da umidade volumétrica do solo. No entanto, apresenta a desvantagem de ser muito sensível à variação de temperatura, salinidade, textura e densidade do solo (BOGENA et al., 2007; SAKAKI et al., 2008; SAITO et al., 2009; ROSENBAUM et al., 2011; SAITO et al., 2013; MENDES et al., 2014; FARES et al., 2015), apresentando também variação de sensor para sensor (ROSENBAUM et al., 2010), o que requer uma calibração individual para cada tipo de solo e para cada sensor.

Assim, neste estudo, objetivou-se avaliar a calibração de um sensor Decagon $\mathrm{ECH}_{2} \mathrm{O}$ EC-5 para um Latossolo do Cerrado, em função da densidade do solo.

\section{MATERIAL E MÉTODOS}

O estudo foi conduzido na área experimental da Universidade Federal de Goiás, localizada no município de Goiânia-GO (16² $1^{\prime}$ S; 4916' W e $741 \mathrm{~m})$. O solo da área experimental foi classificado como um Latossolo Vermelho distrófico, compondo $935.879 \mathrm{~km}^{2}$ do Cerrado brasileiro, ou seja, $46 \%$ da área total (ADÁMOLI et al., 1986). As características químicas e físicas, bem como os parâmetros da curva de retenção (VAN GENUCHTEN, 1980) deste solo, encontram-se nas Tabelas 1 e 2, respectivamente.

Os corpos de prova, para acondicionamento do solo, constituíram-se de cilindros de PVC com diâmetro interno de $0,145 \mathrm{~m}$ e altura de $0,0605 \mathrm{~m}$, 
Tabela 1. Características físico-químicas do solo estudado

\begin{tabular}{|c|c|c|c|c|c|c|c|c|c|c|}
\hline \multirow{2}{*}{$\begin{array}{c}\mathrm{pH} \\
\left(\mathrm{CaCl}_{2}\right)\end{array}$} & $\mathrm{K}$ & $\mathrm{P}$ & $\mathrm{Ca}$ & $\mathrm{Mg}$ & $\mathrm{Al}$ & $\mathrm{H}+\mathrm{Al}$ & \multirow{2}{*}{$\begin{array}{c}\text { M.O. } \\
(\%)\end{array}$} & \multirow{2}{*}{$\begin{array}{c}\text { Areia } \\
(\%)\end{array}$} & \multirow{2}{*}{$\begin{array}{l}\text { Silte } \\
(\%)\end{array}$} & \multirow{2}{*}{$\begin{array}{c}\text { Argila } \\
(\%)\end{array}$} \\
\hline & \multicolumn{2}{|c|}{$--m g ~ d^{-3}--$} & & $---m n$ & $n^{-3}-$ & & & & & \\
\hline 4,7 & 60,0 & 1,7 & 10,0 & 4,0 & 0,0 & 43,0 & 1,5 & 40 & 22 & 38 \\
\hline
\end{tabular}

pH: potencial hidrogeniônico; M.O.: matéria orgânica.

Tabela 2. Parâmetros da curva de retenção de água para o solo em função das distintas densidades

\begin{tabular}{|c|c|c|c|c|c|c|}
\hline $\begin{array}{c}\text { Densidade } \\
\left(\mathrm{Mg} \mathrm{m}^{-3}\right)\end{array}$ & $\begin{array}{c}\theta \mathrm{s} \\
\left(\mathrm{m}^{3} \mathrm{~m}^{-3}\right)\end{array}$ & $\begin{array}{c}\theta c c \\
\left(\mathrm{~m}^{3} \mathrm{~m}^{-3}\right)\end{array}$ & $\begin{array}{c}\theta p m p \\
\left(\mathrm{~m}^{3} \mathrm{~m}^{-3}\right)\end{array}$ & $\begin{array}{c}\alpha \\
\left(\mathrm{kPa}^{-1}\right)\end{array}$ & $\mathrm{m}$ & $\mathrm{n}$ \\
\hline 1,10 & 0,587 & 0,340 & 0,162 & 0,1833 & 0,3633 & 2,0344 \\
\hline 1,20 & 0,572 & 0,382 & 0,167 & 0,2130 & 0,2687 & 1,6803 \\
\hline 1,25 & 0,570 & 0,402 & 0,164 & 0,2017 & 0,2653 & 1,4077 \\
\hline 1,30 & 0,550 & 0,393 & 0,168 & 0,2520 & 0,1981 & 1,6887 \\
\hline 1,35 & 0,554 & 0,399 & 0,136 & 0,4663 & 0,0383 & 5,7257 \\
\hline
\end{tabular}

$\theta$ pmp: umidade referente ao ponto de murcha permanente $(-1500 \mathrm{kPa}) ; \alpha, \mathrm{m}$ e n: parâmetros de ajuste.



Figura 1. Esquema de instalação do sensor Decagon $\mathrm{ECH}_{2} \mathrm{O}$ EC-5 no corpo de prova.

O solo foi seco em estufa a $105{ }^{\circ} \mathrm{C}$ por $24 \mathrm{~h}$. As raízes das plantas e outros materiais indesejados foram removidos das amostras ao passarem por uma peneira de $2 \mathrm{~mm}$.

O solo peneirado foi pesado e adicionado aos corpos de prova nas massas de 1,$00 ; 1,10 ; 1,20$; 1,30 e $1,40 \mathrm{~kg}$. Como o volume dos corpos de prova eram de aproximadamente $0,001 \mathrm{~m}^{3}$, obteve-se assim as densidades de 1,$10 ; 1,20 ; 1,25 ; 1,30$ e 1,35 $\mathrm{Mg} \mathrm{m}^{-3}$, respectivamente. $\mathrm{O}$ solo foi adicionado e comprimido em pequenas quantidades, de forma que as amostras ficassem mais homogêneas possível.

Então, as amostras foram umedecidas por capilaridade até atingirem a saturação, imergindoas em água até $2 / 3$ da altura do corpo de prova. Após 24 horas, foram pesadas em balança, com precisão de $0,01 \mathrm{~g}$, e os valores de umidade volumétrica atual $(\theta a)$ (Equação 1) foram então obtidos:

$$
\theta \mathrm{a}=\left[\frac{(\mathrm{Msu}-\mathrm{Ms})}{\mathrm{Ms}}\right] \mathrm{x} \rho \mathrm{s}
$$

em que,

$\theta \mathrm{a}$ - é a umidade volumétrica atual $\left(\mathrm{m}^{3} \mathrm{~m}^{-3}\right)$;

Msu - é a massa de solo úmida $(\mathrm{kg})$;

Ms - é a massa de solo seco (kg); e

$\rho s$ - é a densidade do solo $\left(\mathrm{Mg} \mathrm{m}^{-3}\right)$.

Iniciou-se então a secagem das amostras em estufa a $40 \quad{ }^{\circ} \mathrm{C}$, em intervalos de 2 horas e espera de 1 hora, para que elas resfriassem à temperatura ambiente, procedendo-se uma nova obtenção dos valores de umidade volumétrica atual. Este procedimento foi repetido até que as amostras atingissem uma umidade volumétrica atual $\leq 0,100 \mathrm{~m}^{3} \mathrm{~m}^{-3}$.

$\mathrm{O}$ ciclo de umedecimento-secagem foi realizado três vezes para cada densidade avaliada, representando assim três repetições.

Neste estudo, o sensor utilizado para obtenção do conteúdo volumétrico de água no solo foi o $\mathrm{ECH}_{2} \mathrm{O}$ EC-5, produzido pela Decagon. Este sensor é do tipo capacitivo e opera à frequência de $70 \mathrm{MHz}$. Ele apresenta dimensões de 0,089 x 0,018 x 0,007 m (Figura 2), o que facilita a sua instalação 
em campo. O corpo do sensor é constituído de uma placa de sílica com a parte sensível (sensor) composto por cobre, com $0,05 \mathrm{~m}$ de comprimento e revestimento em acrílico. O volume de solo medido compreende $0,0003 \mathrm{~m}^{3}$ e o tempo de resposta de cada medição é de aproximadamente 0,2 ms (Decagon Devices Inc., 2016).

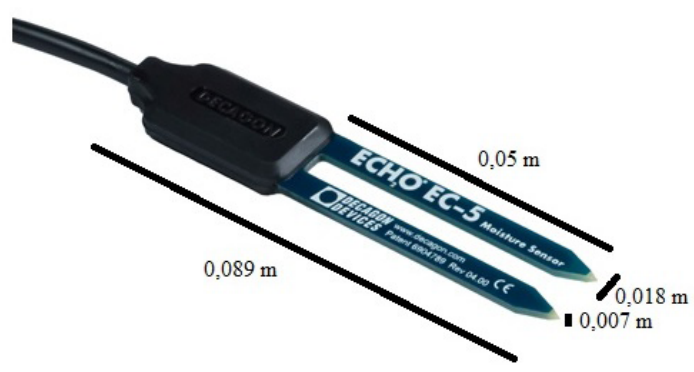

Figura 2. Dimensões do sensor $\mathrm{ECH}_{2} \mathrm{O}$ EC-5.

Os sensores estavam conectados ao datalogger Em50 (Decagon), este, por sua vez, fornece o valor raw (adimensional) que foi obtido simultaneamente à umidade volumétrica atual.

Para facilitar a comparação dos dados, o valor raw, referente a cada sensor, foi convertido em umidade volumétrica estimada, de acordo com a Equação 2, fornecida pelo fabricante:

$\theta 0=8,5 \times 10^{-4} \mathrm{x}$ raw $-0,48$

em que,

$\theta$ o - é a umidade volumétrica observada $\left(\mathrm{m}^{3} \mathrm{~m}^{-3}\right)$; e raw - é o valor fornecido pelo sensor (adimensional).

A avaliação da sensibilidade da calibração dos sensores foi realizada a partir dos seguintes índices estatísticos: índice de concordância $D$ proposto por WILLMOTT (1981); coeficiente de determinação $\left(\mathrm{R}^{2}\right)$; coeficiente residual de massa (CRM) e raiz do erro quadrático médio (REQM), obtidos pelas equações a seguir:

$$
\begin{aligned}
& \mathrm{d}=1-\frac{\sum_{\mathrm{i}=1}^{\mathrm{k}}\left(\hat{\mathrm{y}}_{\mathrm{i}}-\mathrm{y}_{\mathrm{i}}\right)^{2}}{\sum_{\mathrm{i}=1}^{\mathrm{k}}\left(\left|\hat{\mathrm{y}}_{\mathrm{i}}-\overline{\mathrm{y}}\right|+\left|\mathrm{y}_{\mathrm{i}}-\overline{\mathrm{y}}\right|\right)^{2}} \\
& \mathrm{R}^{2}=\left(\frac{\sum_{\mathrm{i}=1}^{\mathrm{k}}\left(\mathrm{x}_{\mathrm{i}}-\overline{\mathrm{x}}\right)\left(\mathrm{y}_{\mathrm{i}}-\overline{\mathrm{y}}\right)}{\sqrt{\sum_{\mathrm{i}=1}^{\mathrm{k}}\left(\mathrm{x}_{\mathrm{i}}-\overline{\mathrm{x}}\right)^{2} \sum_{\mathrm{i}=1}^{\mathrm{k}}\left(\mathrm{y}_{\mathrm{i}}-\overline{\mathrm{y}}\right)^{2}}}\right)^{2}
\end{aligned}
$$

$\mathrm{CRM}=\left[\frac{\sum_{\mathrm{i}=1}^{\mathrm{k}} \hat{\mathrm{y}}_{\mathrm{i}}-\sum_{\mathrm{i}=1}^{\mathrm{k}} \mathrm{y}_{\mathrm{i}}}{\sum_{\mathrm{i}=1}^{\mathrm{k}} \mathrm{y}_{\mathrm{i}}}\right]$

$\operatorname{REQM}=\left[\frac{\sum_{\mathrm{i}=1}^{\mathrm{k}}\left(\hat{\mathrm{y}}_{\mathrm{i}}-\mathrm{y}_{\mathrm{i}}\right)^{2}}{\mathrm{k}}\right]^{1 / 2}$

em que,

$\mathrm{x}_{\mathrm{i}}$ - o valor da umidade volumétrica atual $\left(\theta \mathrm{a}, \mathrm{m}^{3}\right.$ $\left.\mathrm{m}^{-3}\right)$;

$\overline{\mathrm{x}}$ - a média dos valores da umidade volumétrica atual $\left(\theta \mathrm{a}, \mathrm{m}^{3} \mathrm{~m}^{-3}\right)$;

$\mathrm{y}_{\mathrm{i}}$ - o valor da umidade volumétrica estimada $\left(\theta \mathrm{e}, \mathrm{m}^{3} \mathrm{~m}^{-3}\right)$;

$\overline{\mathrm{y}}$ - a média dos valores da umidade volumétrica estimada $\left(\theta \mathrm{e}, \mathrm{m}^{3} \mathrm{~m}^{-3}\right)$;

$\hat{\mathrm{y}}_{\mathrm{i}}$ - os valores estimados da umidade volumétrica atual $\left(\theta \mathrm{a}, \mathrm{m}^{3} \mathrm{~m}^{-3}\right)$; e

k - o número de observações.

Foi adotado o delineamento inteiramente casualizado no esquema fatorial $5 \times 9$. Os fatores de variação avaliados foram as densidades do solo $\left(1,10 ; 1,20 ; 1,25 ; 1,30\right.$ e $\left.1,35 \mathrm{Mg} \mathrm{m}^{-3}\right)$ e as umidades volumétrica observadas $(0,50 ; 0,45 ; 0,40 ; 0,35$; 0,$30 ; 0,25 ; 0,20 ; 0,15$ e $\left.0,10 \mathrm{~m}^{3} \mathrm{~m}^{-3}\right)$, com três repetições, totalizando 135 parcelas experimentais.

Previamente, foi analisada a normalidade e homocedasticidade dos dados pelo teste de ShapiroWil. As variáveis obtidas foram submetidas à análise de variância pelo teste $\mathrm{F}$ à $5 \%$ de probabilidade e suas médias foram submetidas à análise de regressão, à $5 \%$ de significância. Em todos os procedimentos estatísticos descritos foi utilizado o software SISVAR 5.6 (FERREIRA, 2011).

\section{RESULTADOS E DISCUSSÃO}

A umidade volumétrica atual $(\theta a)$ foi superestimada pela calibração fornecida pelo fabricante. Esta tendência foi observada em todas as densidades e para os distintos ciclos de umedecimento-secagem do solo avaliado (Figura 3). Em geral, a superestimativa da $\theta$ a cresceu com o aumento do conteúdo de água no solo acima dos valores de $0,20 \mathrm{~m}^{3} \mathrm{~m}^{-3}$. Para os valores de $\theta \mathrm{a}$ próximos de $0,10 \mathrm{~m}^{3} \mathrm{~m}^{-3}$, a superestimativa ocorreu em menor intensidade. A máxima superestimativa ocorreu para valores de $\theta \mathrm{a}$ acima de $0,30 \mathrm{~m}^{3} \mathrm{~m}^{-3}$. 

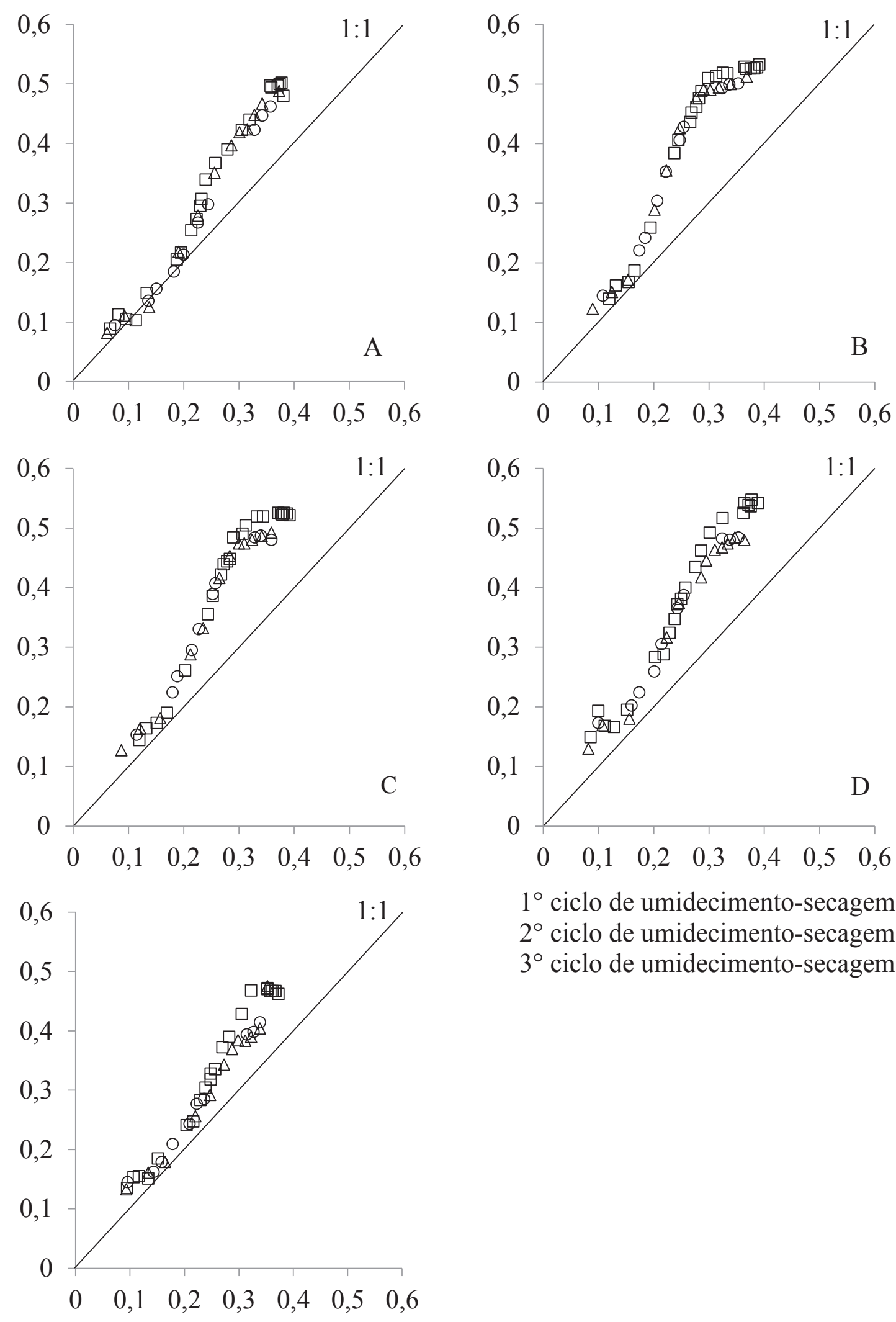

$1^{\circ}$ ciclo de umidecimento-secagem $2^{\circ}$ ciclo de umidecimento-secagem $3^{\circ}$ ciclo de umidecimento-secagem

Umidade volumétrica atual $\left(\theta \mathrm{a}, \mathrm{m}^{3} \mathrm{~m}^{-3}\right)$

Figura 3. Reta 1:1 para a relação entre a umidade volumétrica atual e a observada, utilizando a calibração de fabricação para as densidades de 1,10 (A); 1,20 (B); 1,25 (C); 1,30 (D) e 1,35 (E) $\mathrm{Mg} \mathrm{m}^{-3}$ e o $1^{\circ}$ (quadrado), $2^{\circ}$ (triângulo) e $3^{\circ}$ (círculo) ciclo de umedecimento-secagem. 
De acordo com ROSENBAUM et al. (2010), a Decagon utiliza cinco calibrações padrão: ar $(\varepsilon=$ $1)$, esferas de vidro $(\varepsilon \sim 4,4)$, esferas de vidro com etileno glicol $(\varepsilon \sim 13,5)$, etileno glicol puro $(\varepsilon \sim$ $32,9)$ e água da torneira $(\varepsilon \sim 81,4)$. O objetivo desta calibração é identificar a variabilidade de sensor para sensor e proporcionar uma relação linear entre a resposta do sensor e a permissividade dielétrica (ع) do meio. No entanto, como foi relatado no estudo de DOBSON et al. (1985), a extrapolação da permissividade dielétrica pode ocasionar imprecisão no valor da mesma, que é altamente dependente do tipo de solo.

Desta maneira, quanto menor o conteúdo de água no solo, melhor é a calibração padrão do fabricante, pois, como apresentado anteriormente, em valores de $\theta$ a próximos de $0,10 \mathrm{~m}^{3} \mathrm{~m}^{-3}$, a superestimativa ocorre em menor intensidade, visto que o valor da permissividade dielétrica está mais próximo do real (não extrapolada). Esta tendência também foi observada por SAITO et al. (2009) e FARES et al. (2015), sendo que, no primeiro, o solo era arenoso ( $48,7 \%$ de areia e $44 \%$ de silte) e, no segundo, argiloso ( $70 \%$ de argila).

Conforme os resultados apresentados por DOBSON et al. (1985), a permissividade dielétrica do solo saturado não é a mesma da água pura/ torneira, esta reduz significativamente e varia segundo a textura e estrutura do solo. Então, o que se pode observar é que quando o solo está saturado, ou mesmo, com conteúdo de água mais elevado, a calibração padrão do fabricante se mostra menos eficiente, pois o material utilizado na mesma não extrapola com acurácia a permissividade dielétrica. Calibrações como as deSAITO etal.(2009)eFARES et al. (2015) apresentaram valores subestimados de $\theta \mathrm{a}$, quando comparado a $\theta \mathrm{o}$, mesmo para solos com textura completamente distintas, diferentemente do observado neste estudo, onde a calibração do fabricante superestimou os valores de $\theta$ a. Devido às limitações da calibração padrão do fabricante e a variabilidade nas propriedades do solo, faz-se necessária a calibração dos sensores para cada tipo de solo (FARES et al., 2015).

$\mathrm{Na}$ Tabela 3, são apresentados os valores dos índices estatísticos entre a umidade volumétrica atual e a observada para as diferentes densidades do solo, antes das curvas de calibrações serem realizadas.

Observa-se pela Figura 3 e Tabela 3 que os dados foram superestimados a partir da umidade volumétrica atual de $0,20 \mathrm{~m}^{3} \mathrm{~m}^{-3}$, atingindo valor máximo para valor maior que $0,30 \mathrm{~m}^{3} \mathrm{~m}^{-3}$. Para as densidades de 1,$20 ; 1,25$ e $1,30 \mathrm{Mg} \mathrm{m}^{-3}$, o CRM indica uma superestimativa média dos dados na ordem de 50,$5 ; 44,4$ e $44,7 \%$, respectivamente. Para estas mesmas densidades, a REQM evidencia que a umidade volumétrica pode ser superestimada em 0,$142 ; 0,126$ e $0,120 \mathrm{~m}^{3} \mathrm{~m}^{-3}$. Nestas condições, se o sensor fosse utilizado para o manejo de irrigação sem calibração prévia, poderia informar ao produtor que o solo se encontrava em uma condição de umidade favorável à cultura, sendo que a umidade real estaria próxima ao ponto de murcha permanente.

É possível observar que para a umidade volumétrica atual abaixo de $0,30 \mathrm{~m}^{3} \mathrm{~m}^{-3}$, o ciclo de umedecimento-secagem pouco altera o comportamento da curva de secagem, conforme ilustrado na Figura 3. O primeiro ponto de inflexão, onde apresentam as maiores diferenças nas curvas de secagem, é alterado pelo arranjamento das partículas do solo, devido à presença de poros estruturais. Contudo, com a redução do conteúdo de água no solo, as composições granulométricas e mineralógicas assumem maior importância ao processo de retenção de água no solo (CARDUCCI et al., 2011). Sendo estes os motivos que descrevem a tendência observada durante a calibração do sensor.

$\mathrm{Na}$ Tabela 4, é apresentada a análise de variância da umidade volumétrica estimada. Verifica-se que a interação densidade do solo vs. umidade volumétrica observada apresentou diferença $(\mathrm{P}<0,05)$ para a variável analisada.

A densidade do solo apresentou uma tendência parabólica, sendo que, para as densidades de 1,10 e $1,35 \mathrm{Mg} \mathrm{m}^{-3}$, os valores da umidade volumétrica atual e observada se encontravam mais próximas, ao passo que as densidades intermediárias se encontravam mais distantes da observada, esta tendência pode ser observada pela Figura 4 e Tabela 5. Assim, para determinação da equação de ajuste dos dados, a densidade do solo apresenta-se como equação do segundo grau para melhor ajuste dos dados. 
Tabela 3. Índices estatísticos entre a umidade volumétrica atual e a observada (calibração de fabricação) para as diferentes densidades do solo

\begin{tabular}{cccc}
\hline Densidade $\left(\mathrm{Mg} \mathrm{m}^{-3}\right)$ & $D$ & CRM & REQM $\left(\mathrm{m}^{3} \mathrm{~m}^{-3}\right)$ \\
\hline 1,10 & 0,909 & 0,271 & 0,079 \\
1,20 & 0,722 & 0,505 & 0,142 \\
1,25 & 0,738 & 0,444 & 0,126 \\
1,30 & 0,755 & 0,447 & 0,120 \\
1,35 & 0,875 & 0,254 & 0,068 \\
\hline
\end{tabular}

Os dados apresentados são uma média dos três ciclos de umedecimento-secagem.

Tabela 4. Resumo da análise de variância das variáveis expressa pelo quadrado médio da umidade volumétrica estimada $\left(\mathrm{m}^{3} \mathrm{~m}^{-3}\right)$

\begin{tabular}{lcc}
\hline \multicolumn{1}{r}{ Fator de Variação } & GL & Umidade Volumétrica Estimada \\
\hline DS & 4 & $0,006931^{*}$ \\
OSB & 8 & $0,125275^{*}$ \\
DS*OBS & 32 & $0,000273^{*}$ \\
Resíduo & 90 & 0,000048 \\
\hline & C.V. $(\%)$ & 3,35 \\
\hline
\end{tabular}

*Significativo a 5\% de probabilidade; $\rho$ s: Densidade do solo; OBS: umidade volumétrica observada; C.V.: Coeficiente de variação.

Observa-se pela Figura 4 que a umidade volumétrica pode ser estimada com alto grau de confiabilidade, após a calibração específica para cada densidade do solo ( $\rho s)$, onde o coeficiente de determinação foi igual a 0,9488 . Sendo a equação de ajuste: $\theta \mathrm{e}=0,652376 \theta \mathrm{o}+2,12808{\rho \mathrm{s}^{2}}^{2}-5,2077$ ps $+3,19237$.

$$
\begin{gathered}
\theta \mathrm{e}=0,652376 \theta \mathrm{o}+2,12808 \rho \mathrm{s}^{2}-5,2077 \rho \mathrm{s}+3,19237 \\
\mathrm{R}^{2}=0,9488
\end{gathered}
$$

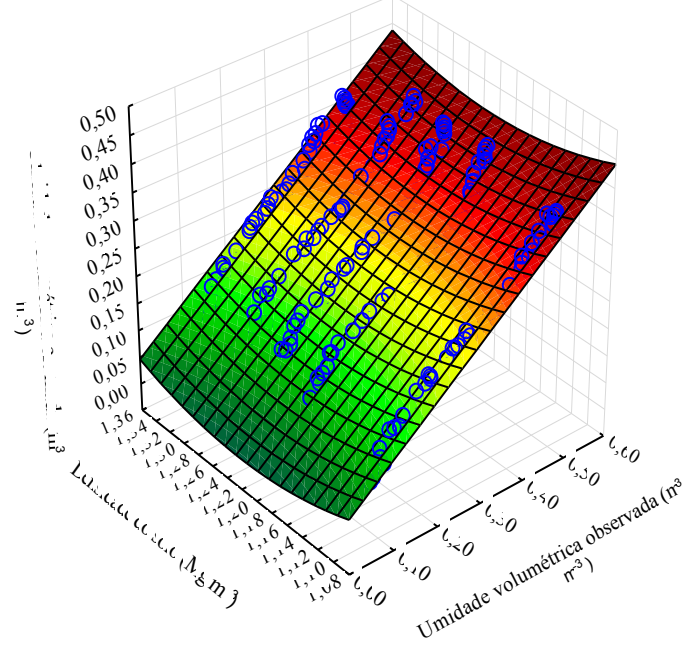

Figura 4. Umidade volumétrica estimada $\left(\theta \mathrm{e}, \mathrm{m}^{3}\right.$ $\mathrm{m}^{-3}$ ) em função da umidade volumétrica observada $\left(\theta \mathrm{o}, \mathrm{m}^{3} \mathrm{~m}^{-3}\right)$ e densidade do solo ( $\rho \mathrm{s}, \mathrm{Mg} \mathrm{m}^{-3}$ ).
$\mathrm{Na}$ Tabela 5, estão presentes os valores dos índices estatísticos entre a umidade volumétrica atual e a estimada para as diferentes densidades do solo, depois de realizada a calibração específica.

Diante dos resultados (Tabela 5), confirmase a acurácia da calibração específica para cada densidade de solo, onde o índice de concordância $D$ foi sempre maior que 0,997 e o CRM muito próximo de 0,00 para todos os casos avaliados, sendo que os valores negativos e positivos indicam subestimativa e superestimativa, respectivamente. A REQM indica uma variação média no valor estimado de $0,019 \mathrm{~m}^{3} \mathrm{~m}^{-3}$, valor este inferior ao especificado pela fabricante como sendo de até $0,030 \mathrm{~m}^{3} \mathrm{~m}^{-3}$, para mais ou para menos.

Na literatura citada no decorrer deste manuscrito, é unânime a opinião de que o sensor EC-5 seja útil e preciso na determinação instantânea da umidade do solo, mas, para tanto, é necessário a correta calibração, principalmente em função da textura e estrutura do solo de interesse. Pois, para cada condição existe uma calibração específica para esse sensor, tal como observado por MENDES et al. (2014), onde, realizando calibração em esterco de galinha, obtiveram uma redução na incerteza da estimativa do conteúdo de água de 30,8 para $7,1 \%$. Contudo, para densidade variando de 0,318 a 0,468 
Tabela 5. Índices estatísticos entre a umidade volumétrica atual e a estimada (calibração específica) para as diferentes densidades do solo

\begin{tabular}{cccc}
\hline Densidade $\left(\mathrm{Mg} \mathrm{m}^{-3}\right)$ & $D$ & CRM & REQM $\left(\mathrm{m}^{3} \mathrm{~m}^{-3}\right)$ \\
\hline 1,10 & 0,998 & $-0,007$ & 0,015 \\
1,20 & 0,999 & 0,011 & 0,024 \\
1,25 & 0,997 & $-0,028$ & 0,020 \\
1,30 & 0,998 & 0,019 & 0,017 \\
1,35 & 0,998 & $-0,015$ & 0,018 \\
\hline
\end{tabular}

Os dados apresentados são uma média dos três ciclos de umedecimento-secagem.

$\mathrm{Mg} \mathrm{m}^{-3}$, foi observado, por esses mesmos autores, um aumento linear da umidade com a mesma, diferentemente do verificado na Figura 4, o que reforça a opinião da necessidade de calibração do sensor EC-5 para interpretação correta dos dados observados.

\section{CONCLUSÕES}

- O sensor EC-5, depois de calibrado, apresenta uma alta acurácia na obtenção do conteúdo de água no solo para o Latossolo Vermelho distrófico.

- Com a inserção da densidade do solo na equação de calibração, houve uma redução média do erro na obtenção do conteúdo de água no solo de $82,24 \%$.

\section{REFERÊNCIA BIBLIOGRÁFICA}

ADÁMOLI, J.; MACEDO, J.; AZEVEDO, L.G.; MADEIRA NETO, J. Caracterização da região dos Cerrados. In: GOEDERT, W.J. Solos dos cerrados: tecnologias e estratégias de manejo. Planaltina: Embrapa-CPAC; São Paulo: Nobel, 1986. p.33-74.

BOGENA, H.R.; HUISMAN, J.A.; OBERDÖRSTER, C.; VEREECKEN, H. Evaluation of a low-coast soil water content sensor for wireless network applications. Journal of Hydrology, Amsterdam, v.344, p.32-42, 2007.

CARDUCCI, C.E.; OLIVEIRA, G.C.; SEVERIANO, E.C.; ZEVIANI, W. M. Modelagem da curva de retenção de água de Latossolos utilizando a Equação Duplo Van Genuchten.
Revista Brasileira de Ciência do Solo, Viçosa, v.35, n.1, p.77-86, 2011.

DECAGON DEVICES INC. EC-5 soil moisture sensor. Operator's manual. Decagon Devices Inc., Pullman, WA. 2016. 22p. Disponível em: <http:// manuals.decagon.com/Manuals/13876_EC-5_ Web.pdf $>$. Acessado em: 28 de setembro de 2016.

DOBSON, M.C.; ULABY, F.T.; HALLIKAINEN, M.T.; EL-RAYES, M.A. Microwave Dielectric Behavior of Wet Soil-Part II: Dielectric Mixing Models. IEEE Transactions on Geoscience and Remote Sensing, Piscataway, v.GE-23, p.35-46, 1985.

FARES, A.; SAFEEQ, M.; AWAL, R.; FARES, S.; DOGAN, A. Temperature and probe-toprobe variability effects on the performance of capacitance soil moisture sensors in an Oxisol. Vadose Zone Journal, Madison, v.3, p.1-40, 2015.

FERREIRA, D.F. Sisvar: a computer statistical analysis system. Ciência e Agrotecnologia, Lavras, v.35, n.6, p.1039-1042, 2011.

MENDES, L.B.; LI, H.; XIN, H.; NASCIMENTO, J.W.B. Evaluation of EC-5 soil moisture sensors for real-time determination of poultry manure or litter moisture contente. American Society of Agricultural and Biological Engineers, St. Joseph, v.30, p.277-284, 2014.

MINET, J.; BOGAERT, P.; VANCLOOSTER, M.; LAMBOT, S. Validation of ground penetrating radar full-waveform inversion for field scale soil moisture mapping. Journal of Hydrology, Amsterdam, v.424-425, p.112-123, 2012. 
ROMANO, N. Soil moisture at local scale: Measurements and simulations. Journal of Hydrology, Amsterdam, v.516, p.6-20, 2014.

ROSENBAUM, U.; HUISMAN, J.A.; VRBA, J.; VERRECKEN, H.; BOGENA, H.R. Correction of temperature and electrical conductivity effects on dielectric permittivity measurements with $\mathrm{ECH}_{2} \mathrm{O}$ sensors. Vadose Zone Journal, Madison, v.10, p.582-593, 2011.

ROSENBAUM,U.; HUISMAN, J.A.; WEUTHEN, A.; VERRECKEN, H.; BOGENA, H.R. Sensorto-sensor variability of the $\mathrm{ECH}_{2} \mathrm{O}$ EC-5, TE e 5TE sensors in dielectric liquids. Vadose Zone Journal, Madison, v.9, p.181-186, 2010.

SU, S.L.; SINGH, D.N.; SHOJAEI BAGHINI, M.A critical review of soil moisture measurement. Measurement, v.54, p.92-105, 2014.

SAITO, T.; FUJIMAKI, H.; YASUDA, H.; INOSAKO, K.; INOUE, M. Calibration of temperature effect on dielectric probes using time serie field data. Vadose Zone Journal, Madison, v.12, p.1-6, 2013.

SAITO, T.; FUJIMAKI, H.; YASUDA, H.; INOUE, M. Empirical temperature calibration of capacitance probes to measure soil water. Soil Science Society of America Journal, Madison, v.73, p.1931-1937, 2009.

SAKAKI, T.; LIMSUWAT, A.; SMITS, K.M.; ILLANGASEKARE, T.H. Empirical two-point $\alpha$-mixing model for calibrating the $\mathrm{ECH}_{2} \mathrm{O}$ EC-5 soil moisture sensor in sands. Water Resources Research, Virginia, v.44, n.4, p.1-8, 2008.

TOPP, G.C; DAVIS, J.L.; ANNAN, A.P. Electromagnetic determination of soil water content: Measurements in coaxial transmission lines. Water Resources Research, Virginia, v.16, p.574-582, 1980.

VAN GENUCHTEN, M.T. A closedform equation for predicting the hydraulic conductivity of unsaturated soils. Soil Science Society of America Journal, Madison, v.44, p.892-898, 1980. 